Acta Crystallographica Section D

Biological

Crystallography

ISSN 0907-4449

\section{Lise Pedersen and Anette Henriksen*}

Carlsberg Laboratory, Department of Chemistry, Gamle Carlsberg Vej 10, DK-2500 Valby, Denmark

Correspondence e-mail: anette@crc.dk

\title{
Expression, purification and crystallization of two peroxisomal acyl-CoA oxidases from Arabidopsis thaliana
}

Two members of the acyl-CoA oxidase family from Arabidopsis thaliana have been cloned, overexpressed, purified and crystallized. Long-chain-specific acyl-CoA oxidase 1 crystals are characterized by a large variation in diffraction quality and non-isomorphous unit-cell parameters. The best crystals diffract to $2.0 \AA$ using synchrotron radiation, have unit-cell parameters $a=85.2, b=117.0, c=131.0 \AA$, $\alpha=\beta=\gamma=90^{\circ}$ and show $P 22_{1} 2_{1} 2_{1}$ symmetry. There are two polypeptide chains in the asymmetric unit. Short-chain-specific acylCoA oxidase 4 crystals are trigonal, space group $P 3_{1} 21 / \mathrm{P}_{2} 21$, with unit-cell parameters $a=b=198.7, c=149.6 \AA$. The crystals are most likely to contain six polypeptide chains in the asymmetric unit. Freshly prepared acyl-CoA oxidase 4 crystals diffract to $3.9 \AA$ at cryogenic temperature at beamline I711, Max-Lab, but the diffraction quality degenerates after storage for only a few days in the crystallization drop. A selenomethionine-substituted form of the protein was produced and two-wavelength MAD data were collected at beamline BW7A, EMBL Outstation, Hamburg.
Received 27 January 2004 Accepted 30 March 2004

\section{Introduction}

Mammals require both a mitochondrial and a peroxisomal system for lipid $\beta$-oxidation (Mannaerts \& Debeer, 1982; Tolbert, 1981), but plants are able to completely degrade fatty acids within the peroxisomes, giving an efficient usage of stored lipids, which is especially beneficial during germination and apoptotic processes (Gerhardt, 1992). Peroxisomal $\beta$-oxidation leads to the formation of acetylCoA through an iterative removal of $\mathrm{C}_{2}$ units from acyl-CoAs (Lazarow \& De Duve, 1976). This process directs fatty acids to gluconeogenesis, although the entire conversion of reserve oil to sucrose after germination involves several pathways and many enzymes within different subcellular compartments (Kindl, 1987). The first and rate-limiting step in peroxisomal fatty-acid $\beta$-oxidation is catalyzed by acyl-CoA oxidases (ACXs; EC 1.3.3.6). These flavin adenine dinucleotide (FAD) containing enzymes are responsible for the conversion of acyl-CoA to trans-2-enoyl-CoA (Hashimoto, 1996). Catalysis is a two-step reaction, the first step being an oxidative introduction of a double bond between $\mathrm{C}$ atoms 2 and 3, resulting in the production of trans-2-enoyl-CoA and reduction of the FAD cofactor. In the oxidative half-reaction, $\mathrm{FADH}^{-}$donates a hydride ion to molecular oxygen, resulting in the formation of $\mathrm{H}_{2} \mathrm{O}_{2}$ (Fig. 1). The ACX family belongs to the same superfamily as the acyl-CoA dehydrogenase family (ACAD; EC 1.3.99.3) that catalyze the equivalent reaction in mitochondria (Kunau et al., 1995). In ACAD the re-oxidation of $\mathrm{FADH}^{-}$is achieved by electron transfer to
(C) 2004 International Union of Crystallography Printed in Denmark - all rights reserved
Reductive half-reaction

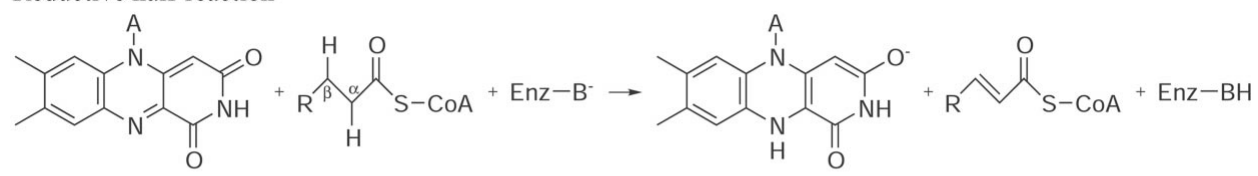

Oxidative half-reaction

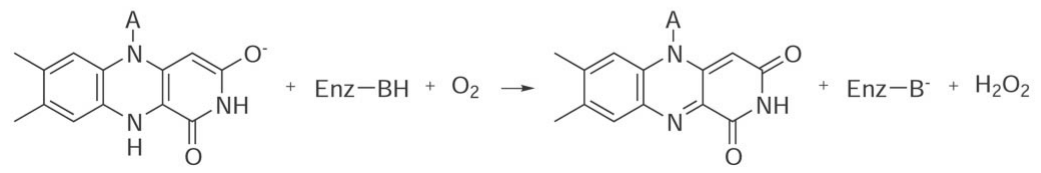

Figure 1

Reaction catalyzed by ACX. 
another flavoprotein, from which the electrons are canalized to the mitochondrial respiratory chain.

Biochemical, physiological and metabolic studies suggest that it is a general property of plants to contain genes that encode several or a family of ACX enzymes (Hayashi et al., 1998; Hooks et al., 1996). Within this ACX family, each isozyme seems to be devoted to the oxidation of acyl-CoAs of specific chain lengths (Kirsch et al., 1987). So far, four different ACXs have been identified in the model oil-seed plant Arabidopsis thaliana (Froman et al., 2000; Hayashi et al., 1999; Hooks et al., 1999).

Presently, we are investigating the structures of $A$. thaliana ACX1 and ACX4. ACX1 has previously been isolated as a homodimer with subunits comprising 664 amino acids and having a molecular weight of $75 \mathrm{kDa}$ (Hooks et al., 1999). It is active on both medium- and long-chain saturated fatty acylCoAs, with maximum activity on $\mathrm{C}_{14}$-CoA (Hooks et al., 1999). ACX4 is a short-chainspecific ACX that shows maximal activity on $\mathrm{C}_{6}$-CoA (Hayashi et al., 2002). Phylogenetic studies show that ACX4 is more closely related to the mitochondrial ACADs than to other ACXs and, like the ACADs, ACX4 has been characterized as a homotetrameric enzyme (Hayashi et al., 1999).

While the enzymology and structural properties of ACADs have been thoroughly investigated (for reviews, see Ghisla \& Thorpe, 2004; Kim \& Miura, 2004), little is known about ACXs and only a single ACX structure has been reported: that of rat ACX (Nakajima et al., 2002). The sequence identities between rat acyl-CoA oxidase and ACX1 and ACX4 are 42 and 12\%, respectively. Further structural studies of different ACX enzymes are needed in order to understand the differences in lipid oxidation between species, the reaction mechanism of this particular class of enzymes, the structural basis of their substrate preferences and the relation to the ACAD-catalyzed reaction.

\section{Experimental}

\subsection{Gene cloning and expression}

The A. thaliana gene encoding ACX1 (Genbank accession No. AY058849) was obtained from the Riken BioResource Center, Japan (clone No. RAFL09-09-K15). The full-length intron-free ACX1 gene was amplified by polymerase chain reaction (PCR) using the primers 5'-CTATCGCCATATGGCGGTGCTTTCATCT-3' and $5^{\prime}$ GCTATCTCGAGCTTGTCGTCGTCGTCG-
AGACGGCTACGTGTACGCGGT-3' and the proof-reading DynaZyme polymerase (Finnzymes). The resultant fragment was cut with restriction enzymes AseI and Not I (New England Biolabs). NdeI and NotI restriction sites were used for cloning the PCR product into the $\mathrm{pET} 24 \mathrm{a}$ expression vector (Novagen), followed by transformation of Escherichia coli BL21(DE3) with the resulting plasmid.

Selected clones were grown aerobically in Luria-Bertani (LB) media containing $50 \mu \mathrm{g} \mathrm{ml}^{-1}$ kanamycin at $310 \mathrm{~K}$ to an $\mathrm{OD}_{600}$ of 0.4. At this point, the cells were cooled on ice until the temperature of the culture reached $277 \mathrm{~K}$. The cells were then incubated at $283 \mathrm{~K}$ and protein expression was induced by the addition of isopropyl- $\beta$-Dthiogalactopyranoside (IPTG) to a final concentration of $0.4 \mathrm{mM}$. Addition of an equal amount of IPTG was repeated at $12 \mathrm{~h}$ intervals over a period of 3-5 d. A yield of $1 \mathrm{mg}$ of soluble ACX1 per litre of cell culture was obtained by cold expression, while conventional $310 \mathrm{~K}$ expression only resulted in the production of insoluble protein. Cells were harvested by centrifugation, resuspended in buffer $A[20 \mathrm{~m} M$ HEPES pH 7.0, $500 \mathrm{~m} M \mathrm{NaCl}, 10 \mu M$ FAD, $0.1 \mathrm{~m} M$ phenylmethylsulfonyl fluoride (PMSF)] and disrupted by sonication. Cell debris was removed by centrifugation at $20000 \mathrm{~g}$ for $60 \mathrm{~min}$. ACX1 was purified from the supernatant by chromatography on a Hi-trap nickel-chelating column (Amersham Biotech) equilibrated in buffer $A$ which, adjusted to $500 \mathrm{~m} M$ imidazole, was used for elution of the protein. After dialysis against buffer $B$ (20 m $M$ HEPES pH 7.0, $150 \mathrm{~m} M$ $\mathrm{NaCl}, 10 \mu M$ FAD) and concentration to a final volume of $5 \mathrm{ml}$, the protein pool was loaded onto an equilibrated Superdex 75 column and eluted at a rate of $0.25 \mathrm{ml} \mathrm{min}^{-1}$. The peak pool was diluted threefold with crystallization buffer ( $20 \mathrm{mM}$ HEPES pH 7.0, $10 \mu M$ FAD) and concentrated to a final concentration of $8 \mathrm{mg} \mathrm{ml}^{-1}$ in a Centriprep YM-30 device (Millipore). The purity of the ACX1 preparation was estimated to be higher than $95 \%$ from an overloaded SDS-PAGE and the final yield of pure recombinant ACX1 was $0.2 \mathrm{mg}$ per litre of cell culture. Gel-filtration experiments indicate dimer-formation of the recombinant His-tagged protein and verification of ACX1 activity was performed using a peroxidase-coupled assay (Gerhardt, 1987) with $25 \mu M$ palmitoyl-CoA as substrate.

The ACX4-encoding gene was amplified by PCR using a cDNA clone (GenBank accession No. T46525) obtained from the
Arabidopsis Biological Resource Center as template. The primers 5'-GAGGCGATTATTATGGAAGGAATTGATCACCTCGCC-3' and 5'-GAATTGCGGCCGCTCAGTGATGGTGATGGTGATGGAGGCTAGCGGTACGAAG-3', where the reverse primer encodes an enterokinase-cleavable C-terminal $6 \times$ His tag, were used for amplification. The amplified DNA was inserted into the pET24a vector using $\mathrm{NdeI}$ and $\mathrm{XhoI}$ restriction sites. The resulting plasmid was transformed into E. coli BL21(DE3) and the cells were grown in LB medium at $310 \mathrm{~K}$. Protein expression was induced at an $\mathrm{OD}_{600}$ of $0.6-0.8$ by the addition of IPTG to a final concentration of $0.5 \mathrm{mM}$. The yield of soluble ACX4 was $15 \mathrm{mg}$ per litre of cell culture after a $3 \mathrm{~h}$ induction period. Harvested cells were resuspended in buffer $A$ and disrupted by ten sonication intervals of $30 \mathrm{~s}$ interspersed with $30 \mathrm{~s}$ intervals without sonication. Cell debris was removed by centrifugation at $20000 \mathrm{~g}$ for $60 \mathrm{~min}$. The supernatant was applied onto a $5 \mathrm{ml} \mathrm{Hi}$-trap Ni-chelating column (Amersham Biosciences) washed with buffer $A$ containing $30 \mathrm{~m} M$ imidazole. The protein was eluted with $1 M$ imidazole in buffer $A$ and dialyzed overnight against buffer $C$ (20 $\mathrm{m} M$ HEPES $\mathrm{pH} 7.8,10 \mu M$ FAD) and concentrated to $10 \mathrm{ml}$, after which ACX4 was purified to homogeneity by size-exclusion chromatography on Superdex-75 eluted at $0.25 \mathrm{ml} \mathrm{min}^{-1}$. Enterokinase (EK) cleavage of ACX4 was performed after the Nichelating affinity chromatography step by incubating ACX4 with EK in a ratio of $20: 1$ at $293 \mathrm{~K}$ for $24 \mathrm{~h}$ while dialyzing against buffer $C$. Cleaved ACX4 was obtained from the flowthough after a second Ni-chelating affinity chromatography step followed by gel-filtration chromatography, yielding $9 \mathrm{mg}$ pure protein per litre of cultured cells.

Incorporation of selenomethionine into ACX4 was obtained by expressing the protein in the B834(DE3) strain deficient in methionine synthesis. The media contained $1 \mathrm{mg} \mathrm{l}^{-1}$ of the vitamins riboflavin, niacinamide, pyridoxine and thiamine, $40 \mathrm{mg} \mathrm{l}^{-1}$ of all amino acids except methionine, $40 \mathrm{mg} \mathrm{l}^{-1}$ seleno-L-methionine, $25 \mathrm{mg} \mathrm{l}^{-1}$ $\mathrm{FeSO}_{4}, 0.4 \%$ glucose, $2 \mathrm{mM} \mathrm{MgSO}$, $2 \mathrm{~g} \mathrm{l}^{-1}$ $\mathrm{NH}_{4} \mathrm{Cl}, \quad 6 \mathrm{~g} \mathrm{l}^{-1} \quad \mathrm{KH}_{2} \mathrm{PO}_{4}, \quad 25.6 \mathrm{~g} \mathrm{l}^{-1}$ $\mathrm{NaPO}_{4} \cdot 7 \mathrm{H}_{2} \mathrm{O}$ and $5 \%$ LB media. Protein expression was induced when the culture reached the exponential stage and was allowed to continue for $8 \mathrm{~h}$. The $\mathrm{C}$-terminal histidine tag was not removed before crystallization because of the lower yields of selenomethionine-substituted ACX4 (5 mg per litre of cell culture). The final yield of 
Table 1

Data-collection statistics.

Values in parentheses are for the last resolution shell.

\begin{tabular}{|c|c|c|c|c|c|}
\hline Data set & $\mathrm{ACX} 1 \mathrm{~K}_{2} \mathrm{PtCl}_{4}$ & $\mathrm{ACX} 1 \mathrm{~K}_{2} \mathrm{PtCl}_{4}$ & ACX4 native & ACX4 inflection & ACX4 peak \\
\hline Beamline & I711, Max-Lab & I711, Max-Lab & I711, Max-Lab & $\begin{array}{l}\text { BW7A, EMBL } \\
\text { Outstation, Hamburg }\end{array}$ & $\begin{array}{l}\text { BW7A, EMBL } \\
\text { Outstation, Hamburg }\end{array}$ \\
\hline Space group & $P 2_{1} 2_{1} 2_{1}$ & $P 2_{1} 2_{1} 2_{1}$ & $P 3_{1} 21 / P 3_{2} 21$ & $P 3_{1} 21 / P 3_{2} 21$ & $P 3_{1} 21 / P 3_{2} 21$ \\
\hline \multicolumn{6}{|l|}{ Unit-cell parameters } \\
\hline$a(\AA)$ & 86.0 & 85.2 & 198.6 & 197.4 & 198.03 \\
\hline$b(\AA)$ & 117.0 & 117.0 & 198.6 & 197.4 & 198.03 \\
\hline$c(\AA)$ & 131.7 & 131.0 & 150.4 & 150.5 & 150.5 \\
\hline Wavelength $(\AA)$ & 1.097 & 1.098 & 1.008 & 0.9783 & 0.9775 \\
\hline Resolution range $(\AA)$ & $30-3.0(3.2-3.0)$ & $40.5-2.0(2.1-2.0)$ & $30-3.9(4.1-3.9)$ & $59.7-4.5(4.7-4.5)$ & $59.7-4.2(4.5-4.2)$ \\
\hline No. unique reflections & 26650 & 88554 & 27647 & 20450 & 29171 \\
\hline Multiplicity & $3.5(2.7)$ & $2.3(2.1)$ & $9.2(9.2)$ & $5.5(5.6)$ & $11.5(11.6)$ \\
\hline Completeness (\%) & $91.8(91.5)$ & $95.8(95.2)$ & $99.8(99.9)$ & $100(100)$ & $100(100)$ \\
\hline$R_{\text {merge }} \dagger$ & $0.15(0.54)$ & $0.11(0.49)$ & $0.15(0.32)$ & $0.28(0.59)$ & $0.37(0.70)$ \\
\hline Mean $I / \sigma(I)$ & $6.4(2.0)$ & $7.4(2.0)$ & $7.4(2.0)$ & $2.4(1.2)$ & $2.2(1.0)$ \\
\hline
\end{tabular}

$\dagger R_{\text {merge }}=\sum_{h k l} \sum_{i}\left|I_{h k l, i}-\left\langle I_{h k l}\right\rangle\right| / \sum_{h k l} \sum_{i} I_{h k l, i}$, where $I$ is the observed intensity and $\langle I\rangle$ is the average intensity for multiple measurements.

pure selenomethionine-substituted ACX4 was $2.0 \mathrm{mg}$ per litre of cell culture.

\subsection{Crystallization}

All crystallization trials were carried out at $293 \mathrm{~K}$ using the hanging-drop vapourdiffusion method (McPherson, 1999). The search for initial crystallization conditions was performed using Hampton Research Crystal Screens I and II (Jancarik \& Kim, 1991) with drops consisting of $2.0 \mu 1$ protein solution at various concentrations mixed with $2.0 \mu \mathrm{l}$ reservoir solution equilibrated against $500 \mu \mathrm{l}$ reservoir solution.

Needle-shaped ACX1 crystals, observed after $1 \mathrm{~d}$ with a protein solution of $8 \mathrm{mg} \mathrm{ml}^{-1}$ and a reservoir consisting of $0.2 \mathrm{M}$ calcium acetate, $0.1 \mathrm{M}$ sodium cacodylate $\mathrm{pH}$ 6.5, 18\% PEG 8000, were optimized by lowering the precipitant concentration to $14-16 \%$ PEG 8000 . The crystals were incubated in mother-liquor solution containing $25 \%$ glycerol for $1 \mathrm{~min}$ for cryoprotection. Several crystals were soaked for 20-30 min in mother liquor

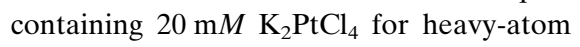
derivatization before cryoprotection and flash-cooling in liquid $\mathrm{N}_{2}$ in a cryoloop (Hampton Research).

ACX4 crystallization resulted in thin needle-like crystals that could be optimized to single hexagonal rods using a reservoir

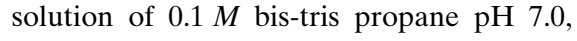
4-8\% PEG 8000 and $6-10 \%$ glycerol. A protein concentration of $2-6 \mathrm{mg} \mathrm{ml}^{-1}$ was used. Prior to mounting the crystals at $100 \mathrm{~K}$, the crystals were transferred stepwise to mother-liquor drops containing 15, 20, 25 and $30 \%$ glycerol.

\subsection{Data collection}

Data sets from two $\mathrm{K}_{2} \mathrm{PtCl}_{4}$-derivative ACX1 crystals were collected at $120 \mathrm{~K}$ using synchrotron radiation at beamline I711, Max-Lab, University of Lund on a MAR CCD detector. The exposure time used was $10 \mathrm{~min}$ per degree of rotation around $\varphi$ and 3 min per degree of rotation around $\varphi$, respectively (Table 1 ).

Data from a native ACX4 crystal were collected at the same beamline, exposing the crystals for 4 min per degree of rotation around $\varphi$, while data at two wavelengths were collected from a selenomethioninesubstituted crystal at beamline BW7A, EMBL Outstation Hamburg, also on a MAR CCD detector. A constant dose was used for the selenomethionine data collection. Processing and scaling were accomplished with MOSFLM (Leslie, 1999) and SCALA (Collaborative Computational Project, Number 4, 1994).

\section{Results and discussion}

ACX1 crystals of dimensions $500 \times 150 \times$ $100 \mu \mathrm{m}$ could be produced within $3-4 \mathrm{~d}$ (Fig. 2a). The crystals are characterized by a large variation in diffraction properties even within the same crystallization drop and

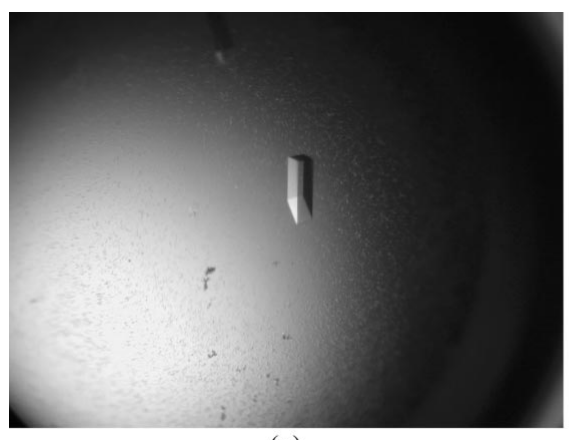

(a)

Figure 2

Crystals of recombinant $A$. thaliana ACXs. (a) ACX1 crystals obtained from $0.2 M$ calcium acetate, $0.1 M$ sodium cacodylate $\mathrm{pH} 6.5$ and 18\% PEG 8000. (b) ACX4 crystals obtained from $0.1 M$ bis-tris propane $\mathrm{pH} 7.0,4 \%$ PEG 8000 and $8 \%$ glycerol. despite there being no apparent differences between the crystals on the macroscopic level. Several cryoprotectants were tested and only addition of $25 \%$ glycerol to the mother liquor proved successful. ACX1 crystals crystallized under identical conditions and cryoprotected in a similar way still showed up to $5.2 \AA$ variation in unit-cell parameters. All crystals belong to space group $P 2_{1} 2_{1} 2_{1}$, with unit-cell parameters $a=85.2, b=117.0, c=131.0 \AA$. The Matthews coefficient $\left(V_{\mathrm{M}}\right)$ of these crystals is $2.23 \AA^{3} \mathrm{Da}^{-1}$, with a corresponding solvent content of $44.5 \%$ assuming two molecules in the asymmetric unit. Two molecular-replacement solutions were obtained from a $3.0 \AA \mathrm{K}_{2} \mathrm{PtCl}_{4}$-derivative data set using the middle and $\mathrm{C}$-terminal domains of the rat acyl-CoA oxidase II structure (Nakajima et al., 2002) as search models in the program MOLREP (Vagin \& Teplyakov, 1997). Rat acyl-CoA oxidase II has $42 \%$ amino-acid sequence identity to $A$. thaliana ACX1. Completion of the structural model was not possible owing to a lack of interpretable density for approximately half of the molecule. The partial ACX1 structure

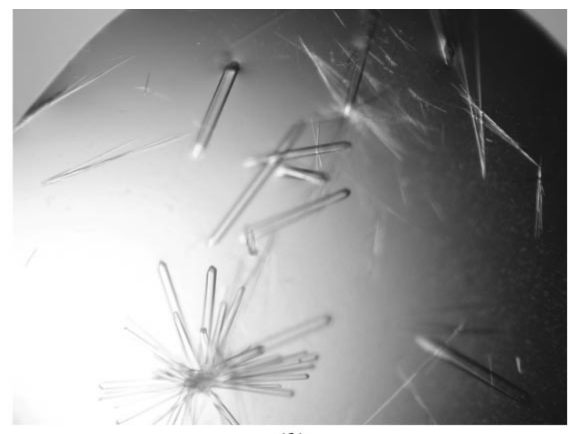

(b) 
was used as search model in AMoRe (Navaza, 1994) against a $2.0 \AA$ platinumderivative data set (using the 12.0-3.5 resolution shell), resulting in two molecularreplacement solutions. After rigid-body refinement of the two molecules in the asymmetric unit, the $R$ factor was 0.485 and the correlation coefficient was 50.5. An initial structural model of 840 residues in 45 fragments was autobuilt using $A R P / w A R P$ (Perrakis et al., 1999). Refinement of the structure is currently in progress.

ACX 4 crystals grew to maximum dimensions of $100 \times 50 \times 50 \mu \mathrm{m}$ within one week (Fig. 2b). Crystals obtained under identical conditions, although similar in size and morphology, exhibited large variations in diffraction quality. No diffraction could be observed if the crystals were kept in the drops for longer than $3 \mathrm{~d}$. Both native and selenomethionine-substituted crystals belong to space group $P 3_{1} 21 / P 3_{2} 21$. Assuming four molecules per asymmetric unit results in a $V_{\mathrm{M}}$ of $4.35 \AA^{3} \mathrm{Da}^{-1}$ and a solvent content of $71.7 \%$; six molecules gives a $V_{\mathrm{M}}$ of $2.48 \AA^{3} \mathrm{Da}^{-1}$ and a solvent content of $50.5 \%$, while eight molecules would result in a $V_{\mathrm{M}}$ of $2.17 \AA^{3} \mathrm{Da}^{-1}$ and a solvent content of $43.4 \%$. The $V_{\mathrm{M}}$ of $2.48 \AA^{3} \mathrm{Da}^{-1}$ for six molecules per asymmetric unit solution best fits the observed $V_{\mathrm{M}}$ distribution in protein crystals (Matthews, 1968). The selfrotation function has only point-groupspecific peaks, but the native Patterson map calculated for 10-4 $\mathrm{A}$ data has a weak but significant $9 \sigma$ translational symmetry peak at $(0.32,0.68,0.61)$ and a $7 \sigma$ peak at $(0.67$, $0.33,0.25)$. Attempts to solve the structure of ACX4 by molecular replacement using the complete structures or domains from medium-chain acyl-CoA dehydrogenase (Kim et al., 1993), buturyl-CoA dehydrogenase (Djordjevic et al., 1995), isovaleryl dehydrogenase (Tiffany et al., 1997) or shortchain acyl-CoA dehydrogenase (Battaile et $a l ., 2002)$ were unsuccessful. The sequence identity between $A$. thaliana ACX4 and the ACADs is only $\sim 20 \%$, while its identity to other ACXs is below 15\%, making the selection of a common structural motif difficult. Production of selenomethioninesubstituted crystals was initiated in order to obtain phase information for ACX4 diffraction. The selenomethionine ACX4 crystals are isomorphous with native ACX4 crystals, making it possible to combine the native data set with the two wavelengths collected on a selenomethionine-substituted crystal for MAD phasing. The low resolution of the selenomethionine data combined with low data quality gives a very weak anomalous signal. This makes the search for the 102 anomalous scatters difficult and additional phase information will be needed in order to solve the structure.

The Danish Natural Research Council (DANSYNC grant) and the European Community ('Access to Research Infrastructure Action of the Improving Human Potential Program' to the EMBL Hamburg Outstation and Max-Lab) supported access to synchrotron sources. We would like to thank the beamline scientists Yngve Cerenius at beamline I711, Max-Lab, Lund and Santosh Panjikar at beamline BW7A, EMBL Hamburg Outstation, who assisted in data collection.

\section{References}

Battaile, K. P., Molin-Case, J., Paschke, R., Wang, M., Bennett, D., Vockley, J. \& Kim, J. J. (2002). J. Biol. Chem. 277, 12200-12207.

Collaborative Computational Project, Number 4 (1994). Acta Cryst. D50, 760-763.

Djordjevic, S., Pace, C. P., Stankovich, M. T. \& Kim, J. J. (1995). Biochemistry, 34, 2163-2171.

Froman, B. E., Edwards, P. C., Bursch, A. G. \& Dehesh, K. (2000). Plant Physiol. 123, 733-742.

Gerhardt, B. (1987). Methods Enzymol. 148, 516525.

Gerhardt, B. (1992). Prog. Lipid Res. 31, 417-446.
Ghisla, S. \& Thorpe, C. (2004). Eur. J. Biochem. 271, 494-508.

Hashimoto, T. (1996). Ann. NY Acad. Sci. 804, 8698.

Hayashi, H., De Bellis, L., Ciurli, A., Kondo, M., Hayashi, M. \& Nishimura, M. (1999). J. Biol. Chem. 274, 12715-12721.

Hayashi, H., De Bellis, L., Hayashi, Y., Nito, K., Kato, A., Hayashi, M., Hara-Nishimura, I. \& Nishimura, M. (2002). Plant Physiol. 130, 20192026.

Hayashi, H., De Bellis, L., Yamaguchi, K., Kato, A., Hayashi, M. \& Nishimura, M. (1998). J. Biol. Chem. 273, 8301-8307.

Hooks, M. A., Bode, K. \& Couee, I. (1996). Biochem. J. 320, 607-614.

Hooks, M. A., Kellas, F. \& Graham, I. A. (1999). Plant J. 20, 1-13.

Jancarik, J. \& Kim, S.-H. (1991). J. Appl. Cryst. 24, 409-411.

Kim, J. J. \& Miura, R. (2004). Eur. J. Biochem. 271, 483-493.

Kim, J. J., Wang, M. \& Paschke, R. (1993). Proc. Natl Acad. Sci. USA, 90, 7523-7527.

Kindl, H. (1987). The Biochemistry of Plants. A Comprehensive Treatise, edited by P. K. Stumpf \& E. E. Conn, pp. 31-52. London: Academic Press.

Kirsch, T., Loffler, H. G. \& Kindl, H. (1987). J. Biol. Chem. 261, 8570-8575.

Kunau, W. H., Dommes, V. \& Schulz, H. (1995). Prog. Lipid Res. 34, 267-342.

Lazarow, P. B. \& De Duve, C. (1976). Proc. Natl Acad. Sci. USA, 73, 2043-2046.

Leslie, A. (1999). MOSFLM. MRC Laboratory of Molecular Biology, Cambridge, England.

McPherson, A. (1999). Crystallization of Biological Macromolecules. New York: Cold Spring Harbor Laboratory Press.

Mannaerts, G. P. \& Debeer, L. J. (1982). Ann. NY Acad. Sci. 386, 30-39.

Matthews, B. W. (1968). J. Mol. Biol. 33, 491-497. Nakajima, Y., Miyahara, I., Hirotsu, K., Nishina, Y., Shiga, K., Setoyama, C., Tamaoki, H. \& Miura, R. (2002). J. Biochem. (Tokyo), 131, 365-374.

Navaza, J. (1994). Acta Cryst. A50, 157-163.

Perrakis, A., Morris, R. M. \& Lamzin, V. S. (1999). Nature Struct. Biol. 6, 458-463.

Tiffany, K. A., Roberts, D. L., Wang, M., Paschke, R., Mohsen, A. W., Vockley, J. \& Kim, J. J. (1997). Biochemistry, 36, 8455-8464.

Tolbert, N. E. (1981). Аnпи. Rev. Biochem. 50, 133-157.

Vagin, A. \& Teplyakov, A. (1997). J. Appl. Cryst. 30, 1022-1025. 